\title{
Dynamical phenomena in Fibonacci semiconductor superlattices
}

\author{
Enrique Diez* \\ Departamento de Matemáticas, Escuela Politécnica Superior, Universidad Carlos III, E-28911 Leganés, Madrid, Spain \\ and Grupo Interdisciplinar de Sistemas Complicados, Escuela Politécnica Superior, Universidad Carlos III, \\ E-28911 Leganés, Madrid, Spain \\ Francisco Domínguez-Adame ${ }^{\dagger}$ \\ Departamento de Física de Materiales, Facultad de Físicas, Universidad Complutense, E-28040 Madrid, Spain; \\ Grupo Interdisciplinar de Sistemas Complicados, Escuela Politécnica Superior, Universidad Carlos III, \\ E-28911 Leganés, Madrid, Spain; \\ and Theoretical Division and Center for Nonlinear Studies, Los Alamos National Laboratory, Los Alamos, New Mexico 87545 \\ Enrique Maciá \\ Departamento de Física de Materiales, Facultad de Físicas, Universidad Complutense, E-28040 Madrid, Spain \\ and Grupo Interdisciplinar de Sistemas Complicados, Escuela Politécnica Superior, Universidad Carlos III, \\ E-28911 Leganés, Madrid, Spain \\ Angel Sánchez ${ }^{\S}$ \\ Departamento de Matemáticas, Escuela Politécnica Superior, Universidad Carlos III, E-28911 Leganés, Madrid, Spain; \\ Grupo Interdisciplinar de Sistemas Complicados, Escuela Politécnica Superior, Universidad Carlos III, \\ E-28911 Leganés, Madrid, Spain; \\ and Theoretical Division and Center for Nonlinear Studies, Los Alamos National Laboratory, Los Alamos, New Mexico 87545
}

(Received 30 April 1996)

\begin{abstract}
We present a detailed study of the dynamics of electronic wave packets in Fibonacci semiconductor superlattices, both in flat band conditions and subject to homogeneous electric fields perpendicular to the layers. Coherent propagation of electrons is described by means of a scalar Hamiltonian using the effective-mass approximation. We have found that an initial Gaussian wave packet is filtered selectively when passing through the superlattice. This means that only those components of the wave packet whose wave numbers belong to allowed subminibands of the fractal-like energy spectrum can propagate over the entire superlattice. The Fourier pattern of the transmitted part of the wave packet presents clear evidences of fractality reproducing those of the underlying energy spectrum. This phenomenon persists even in the presence of unintentional disorder due to growth-induced defects. Finally, we have demonstrated that periodic coherent-field-induced oscillations (Bloch oscillations), which we are able to observe in our simulations of periodic superlattices, are replaced in Fibonacci superlattices by more complex oscillations displaying quasiperiodic signatures, thus shedding more light onto the very peculiar nature of the electronic states in these systems. [S0163-1829(96)08047-2]
\end{abstract}

\section{INTRODUCTION}

Since the fabrication of aperiodic semiconductor superlattices (SL's) arranged according to the Fibonacci ${ }^{1}$ and Thue-Morse $^{2}$ sequences, there has been a growing interest in their electronic properties, both from experimental ${ }^{3-8}$ and theoretical $^{4,9-11}$ viewpoints. One of the most appealing motivations for these studies is the theoretical prediction that ideal aperiodic SL's should exhibit a highly fragmented electronic spectrum displaying self-similar patterns. ${ }^{12,13}$ In fact, low-temperature photoluminescence excitation spectroscopy reveals the existence of a fragmented density of states consistent with theoretical predictions. ${ }^{5}$

Another motivation for the study of dynamical phenomena in aperiodic systems is the following: Electron states in periodic SL's spread uniformly over the whole SL (Bloch states) and the energy spectrum is composed by minibands and minigaps. In the absence of an applied electric field, these extended states are characterized by a transmission probability very close to unity. When a homogeneous electric field is applied perpendicular to the layer plane, electronic states become localized (Stark-Wannier states) and the energy spectrum consists of equally spaced levels (StarkWannier ladder). From the perspective of quantum evolution, Bloch states lead to Bloch oscillations (BO's) when the electric field is applied. ${ }^{14,15}$ The BO period of the electronic motion in real as well as in $k$ space is given by ${ }^{15}$

$$
\tau_{B}=\frac{2 \pi \hbar}{e F d}
$$

where $d$ is the SL constant. High-quality SL's make it possible to obtain $\mathrm{BO}$ periods larger than the scattering time for reasonable values of the applied electric field $F$. Reports of unambiguous experimental evidence for BO's in periodic GaAs-Ga ${ }_{1-y} \mathrm{Al}_{y} \mathrm{As}$ SL's have recently appeared, ${ }^{16,17}$ using 
an experimental method previously proposed by von Plessen and Thomas. ${ }^{18}$ This picture is assumed to be no longer valid in Fibonacci SL's (FSL's) because in the thermodynamical limit electron states are critical instead of extended in flat band conditions, from a strict mathematical point of view. However, since actual FSL's are of finite size, one could expect transport properties similar to those shown by extended electronic states (i.e., high transmission coefficient), since they spread over the whole FSL, although we insist that they are not Bloch states. Hence the question as to whether BO's will be observed or not in FSL arises quite naturally.

Therefore the aim of this work is twofold. In the first place, we provide a complete characterization of electronic states in FSL's, giving a detailed description of dynamical phenomena of electronic wave packets, which, as far as we know, have not been reported in the literature. In this way, we are led to the conclusion that FSL's act as efficient electronic filters, an appealing property in order to use them in actual devices of technological interest. In the second place, we investigate the possibility to observe BO's in FSL's, suggesting the convenience of generalizing the concept of periodic BO's to the case of quasiperiodic oscillations in order to properly describe the dynamical behavior of critical states under the action of homogeneous electric fields.

With the above purpose in mind we numerically solve the effective-mass equation for the envelope function, both in the absence of external fields and under homogeneous electric fields. To be specific, we consider the problem of quantum evolution of electronic wave packets initially localized in space impinging on the FSL. The wave packet dynamics will be properly described by means of the time-dependent transmission probability. The transmitted portion of the wave packet will be characterized by its Fourier transform, aiming to search for particular signatures arising from the scattering event. To get an estimation of the spreading of the wave packet as a function of time, we will use the time-dependent inverse participation ratio (IPR) as well as the mean-square displacement. Finally, since unintentional imperfections appear during fabrication of actual FSL's, we have analyzed a modified version of our model to investigate the possible existence of competition between the long-range quasiperiodic order and the short-range disorder, which could be detected by our time analysis.

\section{MODEL}

We consider quantum well-based $\mathrm{GaAs}^{-\mathrm{Ga}_{1-y}} \mathrm{Al}_{y} \mathrm{As}$ SL's with the same barrier thickness $b$ in the whole sample. The thickness of each quantum well is $\Delta x_{n}-b$ $\equiv x_{n}-x_{n-1}-b, x_{n}$ being the position of the center of the $n$th barrier and $x$ the growth direction. We will focus on electronic states close to the band gap with $\mathbf{k}_{\perp}=0$ and neglect nonparabolicity effects hereafter, so that the Ben Daniel-Duke Hamiltonian suffices to describe those states. The envelope functions for electron wave packets satisfy the following quantum-evolution equation:

$$
i \hbar \frac{\partial \Psi(x, t)}{\partial t}=\mathcal{H}(x) \Psi(x, t) .
$$

The time-independent Hamiltonian $\mathcal{H}(x)$ is given by

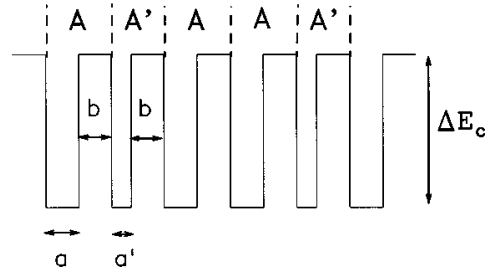

FIG. 1. Schematic diagram of the conduction-band edge of the GaAs-Ga ${ }_{1-y} \mathrm{Al}_{y}$ As Fibonacci superlattice.

$$
\mathcal{H}(x)=-\frac{\hbar^{2}}{2 m^{*}} \frac{d^{2}}{d x^{2}}+V_{\mathrm{SL}}(x)-e F x,
$$

where $V_{\mathrm{SL}}(x)$ is the SL potential under flat band conditions and $F$ is the electric field. The height of the barrier for electrons is given by the conduction-band offset at the interfaces $\Delta E_{c}$. We take the origin of electron energies at the GaAs conduction-band edge.

The particular kind of SL's subject of this work, FSL's, can be grown by means of molecular beam epitaxy, ${ }^{1}$ starting from two basic building blocks $A$ and $A^{\prime}$. Here $A\left(A^{\prime}\right)$ consists of a quantum well of thickness $a\left(a^{\prime}\right)$ and a barrier of thickness $b$. The Fibonacci sequence $S_{n}$ is generated by appending the $n-2$ sequence to the $n-1$ one, i.e., $S_{n}=\left\{S_{n-1} S_{n-2}\right\}$. This construction algorithm requires initial conditions which are chosen to be $S_{0}=A^{\prime}$ and $S_{1}=A$. In this way, finite and self-similar quasiperiodic SL's are obtained by $n$ successive applications of these rules leading to the ordering $A A^{\prime} A A A^{\prime} A \ldots$, containing $N=F_{n}$ barriers. The Fibonacci numbers are generated from the recurrence law $F_{n}=F_{n-1}+F_{n-2}$, starting with $F_{0}=F_{1}=1$. A few blocks of the resulting SL potential $V_{\mathrm{SL}}$ are shown in Fig. 1.

Unintentional disorder appearing during growth in actual SL's depends critically on the growth conditions and it is unknown in most cases. Therefore, one is forced to develop a simple model, making reasonable assumptions on the type of disorder for each particular sample. For instance, islands protruding from one semiconductor into the other cause inplane disorder and break translational invariance parallel to the heterojunction. If the in-plane average size of these protrusions is much larger than the mean free path, then carriers only see an ensemble of different layer thicknesses. ${ }^{19} \mathrm{We}$ model local excess or defect of monolayers by allowing $\Delta x_{n}$ to fluctuate uniformly around the nominal values $a+b$ or $a^{\prime}+b$. For definiteness we take $\Delta x_{n}=a\left(1+W \epsilon_{n}\right)+b$ or $\Delta x_{n}=a^{\prime}\left(1+W \epsilon_{n}\right)+b$, where $W$ is a positive parameter measuring the maximum fluctuation and $\epsilon_{n}$ 's are distributed according to a uniform probability distribution $P\left(\epsilon_{n}\right)=1$ if $\left|\epsilon_{n}\right|<1 / 2$ and zero otherwise. Note that $\epsilon_{n}$ is a random variable, even when the mean values of $\Delta x_{n}$ follow the Fibonacci sequence.

\section{NUMERICAL ANALYSIS}

We study the quantum dynamics of an initial Gaussian wave packet,

$$
\Psi(x, 0)=\left[2 \pi(\Delta X)^{2}\right]^{-1 / 4} \exp \left[\frac{i k_{0} x-\left(x-x_{0}\right)^{2}}{4(\Delta X)^{2}}\right],
$$


impinging on the FSL, where the mean kinetic energy is $\langle E\rangle=\hbar^{2} k_{0}^{2} / 2 m^{*}$ and $\Delta X$ measures the width of the electron wave packet. The solution of Eq. (2) is given by

$$
\Psi(x, t)=\exp \left(-\frac{i}{\hbar} \mathcal{H}(x) t\right) \Psi(x, 0) .
$$

The finite difference representation of the exponential ${ }^{21}$

$$
\exp \left(-\frac{i}{\hbar} \mathcal{H}(x) \delta t\right)=\frac{1-\frac{i}{2 \hbar} \mathcal{H}(x) \delta t}{1+\frac{i}{2 \hbar} \mathcal{H}(x) \delta t}+\mathcal{O}\left[(\delta t)^{3}\right],
$$

where $\delta t$ is the time step, provides a powerful and highly accurate numerical method. In addition, it ensures probability conservation, ${ }^{22}$ which has been used at every time step as a first test of the accuracy of results. Boundary conditions $\operatorname{read} \Psi(\infty, t)=\Psi(-\infty, t)=0$ and we have chosen the FSL sufficiently large to be sure that the wave packet never comes close to the boundaries.

Transmission properties of the electronic wave packet can be successfully analyzed by means of the time-dependent transmission probability $P_{T}(t)$, which is nothing but the probability that at time $t$ the electron is found to have crossed the whole SL,

$$
P_{T}(t)=\int_{L}^{\infty} d x|\Psi(x, t)|^{2},
$$

where $L$ is the length of the system. In addition, to get a complete characterization of the motion of the wave packet, we use the time-dependent inverse participation ratio, $\operatorname{IPR}(t)$, and the mean-square displacement, $\sigma(t)$. The IPR is defined as

$$
\operatorname{IPR}(t)=\int_{-\infty}^{\infty} d x|\Psi(x, t)|^{4},
$$

and it gives an estimation of the spatial extent and the degree of localization of electronic wave packets, which can indeed provide very much information. Delocalized states are expected to present small IPR (in the ballistic limit, without applied field, it vanishes as $t^{-1}$ ), while localized states have larger IPR. The mean-square displacement describes how quantum diffusion of wave packets initially located in the middle of the FSL takes place. The mean-square displacement $\sigma(t)$ is defined as

$$
\sigma^{2}(t)=\int_{-\infty}^{\infty}(x-\bar{x})^{2}|\psi(x, t)|^{2} d x
$$

with

$$
\bar{x}=\int_{-\infty}^{\infty} x|\psi(x, t)|^{2} d x
$$

In the asymptotic regime $(t \rightarrow \infty)$ one expects $\sigma^{2}(t) \sim t^{\gamma}$. The exponent is $0<\gamma<1$ for localized states, $\gamma=1$ for ordinary diffusion, $1<\gamma<2$ for superdiffusion, and $\gamma=2$ for the ballistic regime.

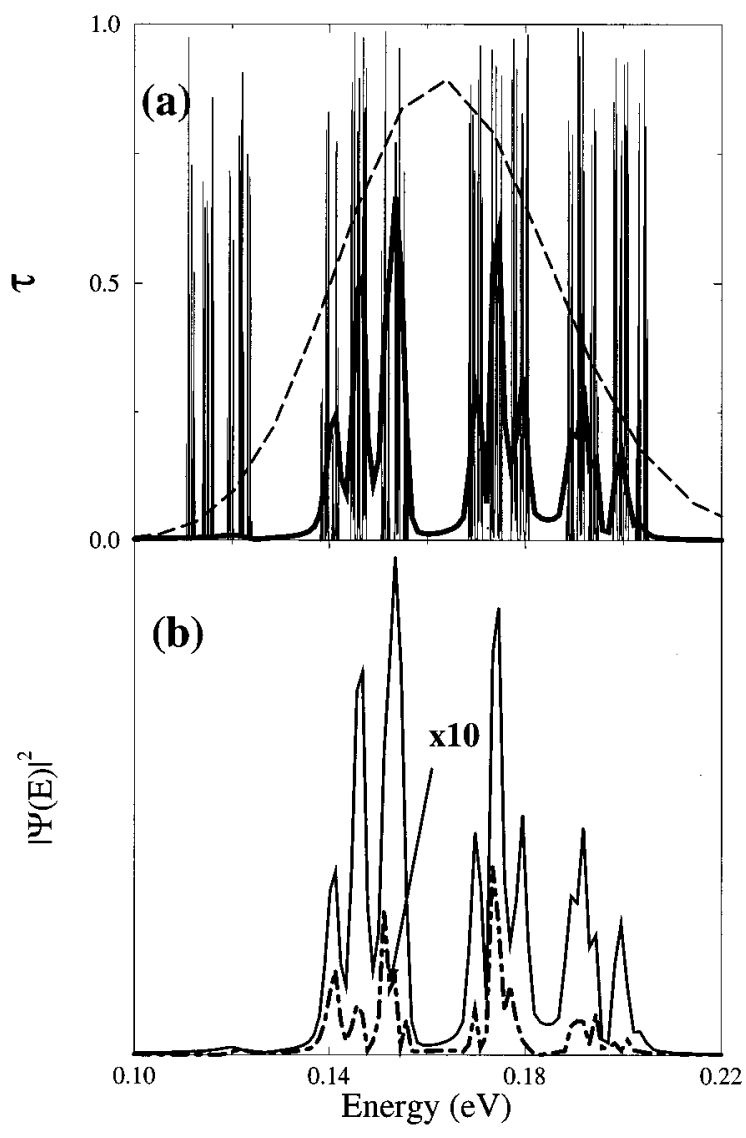

FIG. 2. (a) Transmission coefficient versus incoming energy for a GaAs- $\mathrm{Ga}_{065} \mathrm{Al}_{035} \mathrm{As}$ FSL with $N=144$ wells (thin solid line). Fourier transforms of the initial wave packet $(\Delta X=200 \AA$ and $\langle E\rangle=0.160 \mathrm{eV}$, dashed line) and the transmitted wave packet for perfect FSL ( $W=0$, thick solid line) at $t=6$ ps are also shown in arbitrary units. (b) Fourier transforms of the trasmitted wave packets for a perfect ( $W=0$, solid line) and imperfect ( $W=0.05$, dashed line) FSL's at $t=6 \mathrm{ps}$. Notice in (a) the perfect coincidence between the filtered wave packet and the allowed minibands. Parameters are $a=b=32 \AA$ and $a^{\prime}=26 \AA$.

\section{RESULTS AND DISCUSSIONS}

\section{A. Zero field behavior}

As a typical SL we have chosen a GaAs- $\mathrm{Ga}_{06} \mathrm{Al}_{035} \mathrm{As}$ structure, for which the conduction-band offset is $\Delta E_{c}=0.25 \mathrm{eV}$ and the effective-mass is $m^{*}=0.067 \mathrm{~m}, \mathrm{~m}$ being the free electron mass. In our computations we have taken $a=b=32 \AA$ and $a^{\prime}=26 \AA$. With these parameters there exists only one miniband below the barrier in periodic SL's (built of A's only), ranging from $0.102 \mathrm{eV}$ up to $0.177 \mathrm{eV}$. Thus the miniband width is much larger than the exciton binding energy, which amounts $\sim 0.01 \mathrm{eV}$ in the present SL's. This is a relevant fact, for it has been shown that electronic localization (Stark-Wannier states) is suppressed for low miniband widths. ${ }^{23}$ Finally, to compare with actual SL's, we have considered the parameter $W$, which governs the imperfection magnitude, ranging from 0 up to a maximum of 0.05 . This value amounts to having defect thicknesses of half a monolayer on average.

Figure 2 shows the results of a typical simulation of a wave packet for a FSL. First, in Fig. 2(a) we show the trans- 
mission coefficient, $\tau$, as a function of energy for a perfect ( $W=0)$ FSL with $N=144$ wells, computed by means of the transfer-matrix formalism. ${ }^{3,8,24}$ The overall structure of the energy spectrum is characterized by the presence of four main subminibands (notice that transmission peaks are clustered around energies $\sim 0.120,0.155,0.175$, and $0.200 \mathrm{eV}$ ). An enlarged view of each cluster of peaks shows that the fragmentation pattern follows a trifurcation scheme in which each cluster splits from one to three subclusters upon going to higher-order generations of FSL's. This splitting scheme agrees with that previously reported in FLS's. ${ }^{9,25}$ The main question is to know how this highly fragmented energy spectrum affects the quantum evolution of a wave packet incident on the FSL.

Figure 2(a) shows also the Fourier transform of an initial Gaussian wave packet of width $\Delta X=200 \AA$ at $t=0$ with its average kinetic energy $\langle E\rangle=0.160 \mathrm{eV}$ lying in the centermost subminigap. After the transmitted electron is found to have crossed the whole FSL [ $\sim 6$ ps, see Fig. 3(a)], the Fourier transform of the electronic wave packet changes dramatically in perfect FSL's $(W=0)$. Instead of a smooth function, the Fourier transform presents a series of marked peaks. Conspicuously, the energy of these peaks coincides with the higher values of the transmission coefficient, thus indicating that the FSL acts as an efficient electronic filter. Notice that the Fourier transform also displays the same splitting pattern as the energy spectrum, being observable even at the third level of hierarchy in the upper subminiband. A physical understanding of this behavior is achieved if one considers that the initially localized wave packet can be regarded as a combination of plane waves in a continuous band. Since the dispersion relation (energy versus wave number) is self-similar with a hierarchy of split subminibands separated by welldefined minigaps [see the transmission coefficient shown in Fig. 2(a)], only those components whose wave number belongs to an allowed subminiband can propagate over large distances and, consequently, contribute to the transmitted part of the wave packet. What is most important for practical purposes, we have found that unintentional disorder does not severely affect filtering properties, as shown in Fig. 2(b) for $W=0.05$. Although an overall reduction of the transmitted components is seen, signatures of the above-mentioned level splitting are still clearly observed in the Fourier pattern, particularly at the central energy region around $0.155 \mathrm{eV}$.

Quantum evolution of electronic wave packets will depend upon the system length since the fragmentation of the energy spectrum is higher on increasing $N$. Figure 3(a) shows the time-dependent transmission probability as a function of time for perfect ( $W=0$ ) FSL's of various lengths. For comparison, it should be kept in mind that the transmission probability vanishes in intentionally disordered SL's for moderately large sizes, thus providing further evidence of the differences between random and aperiodic systems. The occurrence of the plateau for larger times indicates that the transmitted wave packet has crossed the whole FSL. Thus, the larger the FSL, the later the plateau appears, as was to be expected. Interestingly, the value of the asymptotic probability decreases upon increasing the FSL length. This reduction of the transmission probability means that filtering effects are stronger as the fragmentation of the energy spectrum is higher. That is to say, the equivalent miniband width, defined
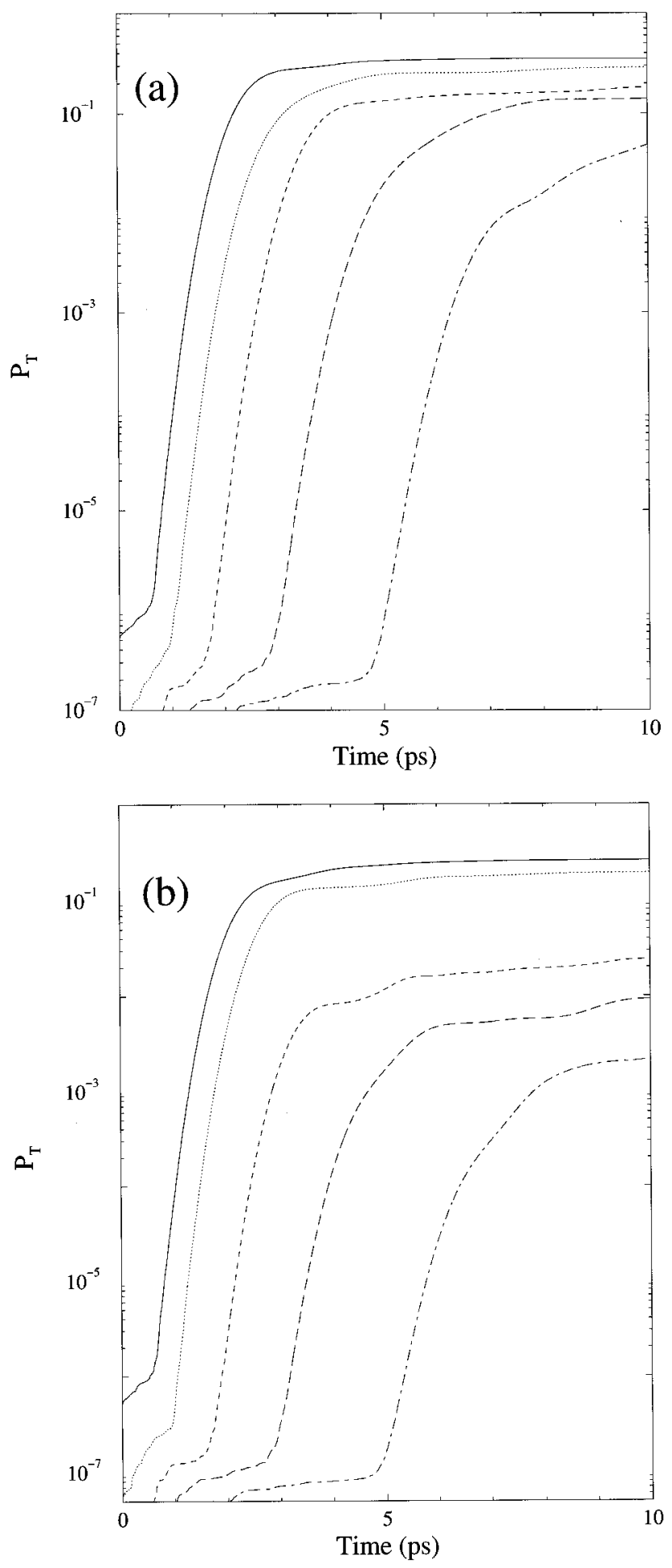

FIG. 3. Transmission probability versus time for (a) perfect $W=0$ and (b) imperfect $W=0.05$ GaAs- $\mathrm{Ga}_{06}{ }_{65} \mathrm{Al}_{035} \mathrm{As}$ FSL with various numbers of wells: From top to bottom, $N=34,55,89,144,233$. Other parameters are the same as in Fig. 2.

as the sum of all the allowed subminibands, decreases as a power of $N$ as a consequence of the quasiperiodic topology of the FSL (Ref. 11) and, therefore, more and more components of the wave packet are back reflected. Figure 3(b) presents the results for the time-dependent transmission probability as a function of time for imperfect ( $W=0.05)$ FSL's of various lengths. A comparison with Fig. 3(a) indicates a 


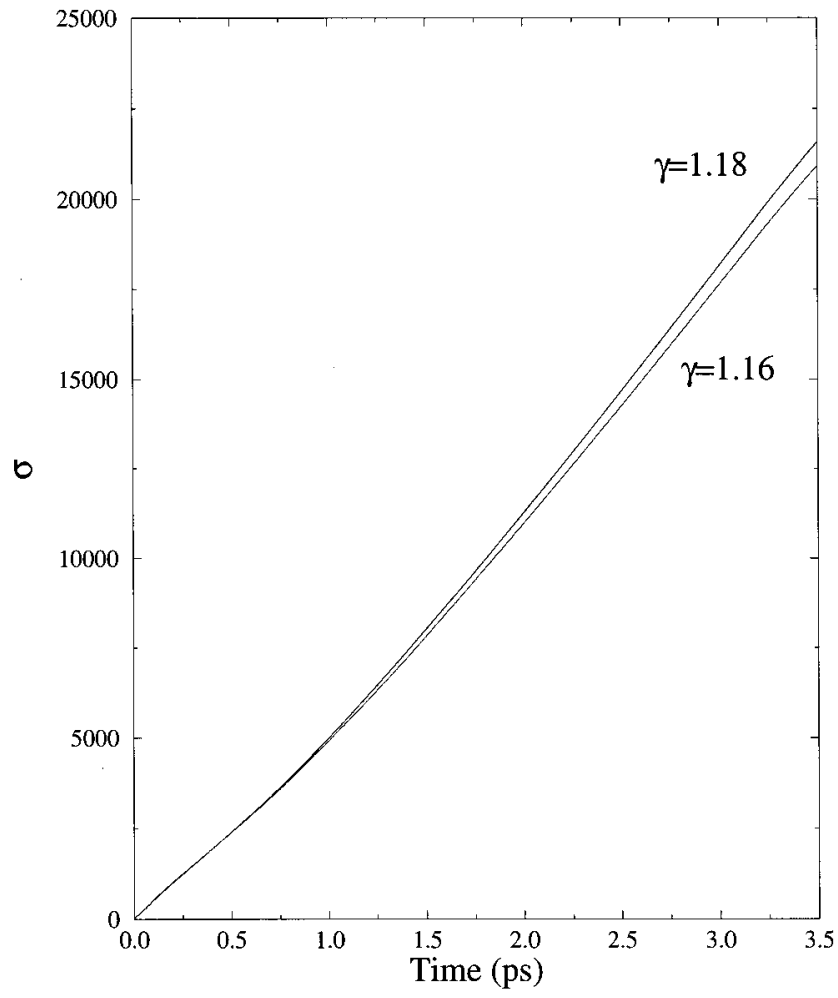

FIG. 4. Mean-square displacement versus time for perfect ( $W=0$, solid line) and imperfect ( $W=0.05$, dashed line) GaAs$\mathrm{Ga}_{065} \mathrm{Al}_{035} \mathrm{As}$ FSL with $N=377$ wells. Other parameters are the same as in Fig. 2. The mean-square displacement grows in time as a power law $\sim t^{\gamma}$. $\sigma$ is measured in $\AA$.

decrease of the transmission probability since short-range disorder tends to localize particles, according to Anderson theory. However, this effect is almost unnoticeable for relatively short (say $N$ smaller than 89) FSL, in agreement with our previous estimations based on the study of the equivalent miniband width. ${ }^{26}$ Therefore, we are led to the conclusion that moderately large fluctuations cannot destroy filtering effects of actual FSL's and that they can safely be used for such purposes. The study of the mean-square displacement in perfect and imperfect FSL's provides additional support in this sense. We have placed the initial Gaussian wave packet in the middle of the FSL, with $\Delta X=20 \AA$ and $\langle E\rangle=0.160 \mathrm{eV}$. In all cases we have observed the super diffusive regime $\sigma^{2}(t) \sim t^{\gamma}$ with $\gamma \simeq 1.2$, as illustrated in Fig. 4 . This result shows that the time-dependent properties are quite similar for both perfect and imperfect FSL's as large as samples containing $N=377$ wells. It is also interesting to note that the exponent $\gamma$ obtained in our study, which is based on a realistic continuous model, is lower than that obtained by considering tight-binding Hamiltonians. ${ }^{27}$

\section{B. Homogeneous field effects}

We now comment on our results in the situation when a homogeneous electric field is applied perpendicular to the layers. One expects that BO's are to be observed in periodic SL's in the limit of high electric fields. The localization length of Stark-Wannier states, the static counterpart of the BO's, is of the order of $\Delta E / e F, \Delta E$ being the width of the
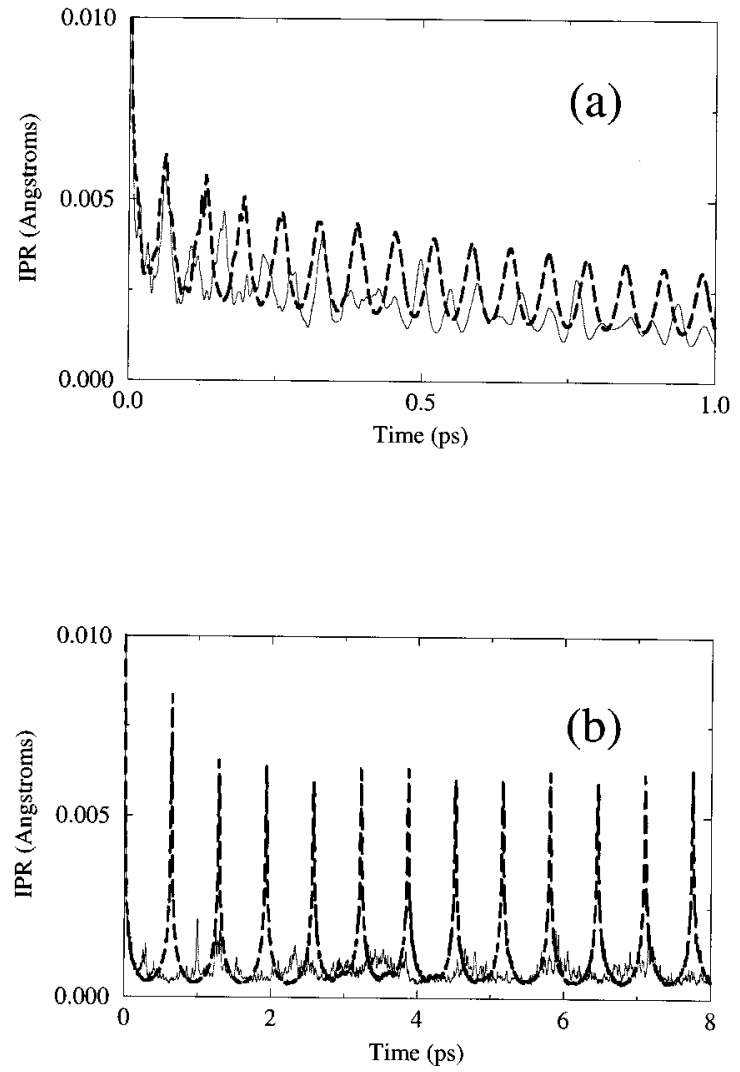

FIG. 5. Inverse participation ratio as a function of time for an initial Gaussian wave packet placed in periodic (dashed line) and Fibonacci (solid line) GaAs- $\mathrm{Ga}_{06}{ }_{65} \mathrm{Al}_{0}{ }_{35} \mathrm{As}$ SL's, subject to an electric field (a) $F=100 \mathrm{kV} / \mathrm{cm}$ and (b) $10 \mathrm{kV} / \mathrm{cm}$. Other parameters are the same as in Fig. 2. The occurrence of Bloch oscillations in the periodic SL is apparent.

allowed miniband, so that we can roughly estimate that the condition $\Delta E / e F \sim a+b$ establishes the high-field regime. In our SL this threshold field is $F \simeq 100 \mathrm{kV} / \mathrm{cm}$. Figure 5 (a) presents the results for the IPR when the initial Gaussian wave packet with $\Delta X=20 \AA$ and $\langle E\rangle=0.160 \mathrm{eV}$ is located in the middle of the periodic SL, the electric field being $F=100 \mathrm{kV} / \mathrm{cm}$. The IPR displays periodic oscillations with marked peaks at times $t_{k}=k \tau_{B}$, where $k$ is any arbitrary, nonnegative integer and $\tau_{B}=0.065 \mathrm{ps}$. Thus the IPR is bounded below, indicating that the wave packet is localized (dynamical localization) but its spatial extent varies periodically in time. Notice that the value of the oscillation period is in excellent agreement with the theoretical prediction $\tau_{B}=2 \pi \hbar /(e F d)$ given in Eq. (1). It is also worth mentioning that the initial state $\Psi(x, 0)$ is not completely restored after each oscillation, as should be if interminiband tunneling effects were negligible. ${ }^{18}$ Thus we are led to the conclusion that interminiband tunneling plays a role in our periodic SL.

Results corresponding to a FSL with the same initial condition as before are also shown in Fig. 5(a). First of all, we observe that at short times we can detect two oscillations coinciding with the positions of the first two BO peaks, but at larger times periodic BO's are completely absent in FSL's. The absence of Bloch oscillations in FSL's simply reflects the fact that their extended states are no longer Bloch states. Bloch states are characterized by a periodic pattern, 
but this is not the case in the FSL, where critical states spreading over the whole system show quasiperiodic patterns. ${ }^{20}$ In this sense, signatures of a quasiperiodic oscillation pattern in the IPR corresponding to the FSL can be seen from Fig. 5(a) in the interval $0.5 \leqslant t \leqslant 1$ ps. To this end, we introduce the ratio $p / q$, where $q$ is the number of oscillations of the FSL IPR comprised in a given number $p$ of periodic BO's. In this way we obtain the following sequence $p / q=2 / 3,3 / 5,2 / 3,5 / 8,2 / 3,7 / 11,2 / 3 \ldots$, which converges to $2 / 3$, an approximant of the inverse golden mean $\tau_{G} \equiv(\sqrt{5}-1) / 2$.

This fact can be described by means of the following scenario, based on our previous discussion on the localization degree of electronic states under an applied electric field. For such high electric fields the spatial extent of the electronic states is of the order of the SL constant and, consequently, the electron cannot see the long-range quasiperiodic potential. In fact, differences between periodic and Fibonacci SL's at short times are actually very small [see Fig. 5(a)]. As soon as some components escape from the localization region (for instance, those states above the barrier, which have a large transmission coefficient) the effect of quasiperiodicity appears. To get further confirmation of this interpretation we have also studied the case of lower electric fields, as shown in Fig. 5(b) for $F=10 \mathrm{kV} / \mathrm{cm}$, when the localization length is about ten SL periods. It becomes apparent that no signatures of BO's can be detected even at short times. Moreover, Fig. 5(b) also displays the result for the periodic SL and the same electric field. Notice that the initial state is completely restored after each BO, suggesting that in this regime interband tunneling is actually negligible.

\section{CONCLUSION}

In this paper we have studied quantum dynamics of wave packets in Fibonacci SL's. After solving numerically the time-dependent effective-mass equation arising from the Ben Daniel-Duke Hamiltonian, we have found that an initial Gaussian wave packet undergoes complicated scattering events at the FSL. In particular, the Fourier spectrum of the transmitted part of the wave packet is no longer smooth but presents many marked peaks. These peaks correspond to the allowed subminibands of the energy spectrum, thus being a clear indication that the FSL acts as a good electronic filter. Transmission properties under zero bias have also been successfully characterized by means of the transmission prob- ability given by Eq. (7). From these results we have learned that unintentional disorder arising during the growth processes have no noticeable effects on the filtering capabilities of short FSL's, because they only cause an overall decrease of the transmitted amplitude. On the other hand, the spatial degree of localization of wave packets driven by an electric field has been properly described by means of the timedependent IPR: As a check, in periodic SL's we have obtained the dynamical localization fields as well as BO's. On the other hand, quantum dynamics in FSL's also exhibits dynamical localization although it turns out to be much more intricate. In particular, no evidence of periodic Bloch oscillations was observed. Instead, the very concept of BO should be changed in order to include the possibility of quasiperiodic pattern oscillations emerging from the quasiperiodic nature of the system. This result adds another piece of evidence about the very peculiar nature of electronic states in Fibonacci systems. ${ }^{20}$

As a concluding remark, we want to stress that with this work we have sufficiently demonstrated the existence of distinct physical observable consequences of systems with singular energy spectra such as those of the FSL. For instance, the fragmentation of the SL minibands could serve as a basis for electronic filters, in a similar fashion to metallic Fibonacci multilayers acting as selective filters of soft x-ray radiation..$^{28}$ It is quite clear that the output of such a device is but an image of its fractal spectrum, which, in turn, is intimately connected with the quasiperiodic nature of the SL and hence with the information it contains. Therefore, aside from the possibility of building the specific filterlike devices we already mentioned, designed with this or other quasiperiodic sequence according to the desired application, it might as well be that this kind of system can be used in transmitting or processing information. These and other relations already established between quasiperiodic nanoelectronic devices and information theory ${ }^{25}$ pave the way to a very exciting and promising line of cross-disciplinary research.

\section{ACKNOWLEDGMENTS}

F. D.-A. and A. S. are thankful to Alan R. Bishop for the warm hospitality enjoyed during their stay at Los Alamos. Work at Madrid and Leganés has been supported by CICYT (Spain) under project No. MAT95-0325. Work at Los Alamos is performed under the auspices of the U.S. DOE.
*Electronic address: diez@dulcinea.uc3m.es

†Electronic address: adame@valbuena.fis.ucm.es

†Electronic address: macia@valbuena.fis.ucm.es

${ }^{\S}$ Electronic address: anxo@dulcinea.uc3m.es

${ }^{1}$ R. Merlin, K. Bajema, R. Clarke, F.-Y. Juang, and P. Bhattacharya, Phys. Rev. Lett. 55, 1768 (1985).

${ }^{2}$ R. Merlin, K. Bajema, J. Nagle, and K. Ploog, J. Phys. (France) Colloq. 48, C5-503 (1987).

${ }^{3}$ R. Merlin, IEEE J. Quantum Electron. 24, 1791 (1988).

${ }^{4}$ F. Laruelle and B. Etienne, Phys. Rev. B 37, 4816 (1988).

${ }^{5}$ A. A. Yamaguchi, T. Saiki, T. Tada, T. Ninomiya, K. Misawa, and T. Kobayashi, Solid State Commun. 75, 955 (1990).

${ }^{6}$ D. Toet, M. Potemski, Y. Y. Wang, J. C. Maan, L. Tapfer, and K.
Ploog, Phys. Rev. Lett. 66, 2128 (1991).

${ }^{7}$ K. Hirose, D. Y. K. Ko, and H. Kamimura, J. Phys. Condens. Matter 4, 5947 (1992).

${ }^{8}$ D. Munzar, L. Bočáek, J. Humlíček, and K. Ploog, J. Phys. Condens. Matter 6, 4107 (1994).

${ }^{9}$ E. Maciá, F. Domínguez-Adame, and A. Sánchez, Phys. Rev. B 49, 9503 (1994).

${ }^{10}$ F. Domínguez-Adame, E. Maciá, and B. Méndez, Phys. Lett. A 194, 184 (1994).

${ }^{11}$ F. Domínguez-Adame, E. Maciá, B. Méndez, C. L. Roy, and A. Khan, Semicond. Sci. Technol. 10, 797 (1995).

${ }^{12}$ M. Kohmoto, L. P. Kadanoff, and C. Tang, Phys. Rev. Lett. 50, 1870 (1983). 
${ }^{13}$ C. S. Ryu, G. Y. Oh, and M. H. Lee, Phys. Rev. B 48, 132 (1993).

${ }^{14}$ F. Bloch, Z. Phys. 52, 555 (1928); C. Zener, Proc. R. Soc. London Ser. A 145, 523 (1934).

${ }^{15}$ M. Dignam, J. E. Sipe, and J. Shah, Phys. Rev. B 49, 10502 (1994).

${ }^{16}$ K. Leo, P. Haring, F. Brüggemann, R. Schwedler, and K. Köhler, Solid State Commun. 84, 943 (1992).

${ }^{17}$ J. Feldmann, K. Leo, J. Shah, D. A. B. Miller, J. E. Cunningham, T. Meier, G. von Plessen, A. Schulze, P. Thomas, and S. Schmitt-Rink, Phys. Rev. B 46, 7252 (1992).

${ }^{18}$ G. von Plessen and P. Thomas, Phys. Rev. B 45, 9185 (1992).

${ }^{19}$ K. A. Mäder, L. -W. Wang, and A. Zunger, J. Appl. Phys. 78, 6639 (1995).

${ }^{20}$ E. Maciá and F. Domínguez-Adame, Phys. Rev. Lett. 76, 2957 (1996).

${ }^{21}$ See W. H. Press, B. P. Flannery, S. A. Teukolsky, and W. T.
Wetterling, Numerical Recipes (Cambridge University Press, New York, 1986).

${ }^{22}$ A. M. Bouchard and M. Luban, Phys. Rev. B 52, 5105 (1995).

${ }^{23}$ P. Leisching, P. Haring Bolivar, W. Beck, Y. Dhaibi, F. Brüggemann, R. Schwedler, H. Kurz, K. Leo, and K. Köhler, Phys. Rev. B 50, 14389 (1994).

${ }^{24}$ F. Domínguez-Adame, A. Sánchez, and E. Diez, Phys. Rev. B 50, 17736 (1994).

${ }^{25}$ E. Maciá, F. Domínguez-Adame, and A. Sánchez, Phys. Rev. E 50, R679 (1994).

${ }^{26}$ E. Maciá and F. Domínguez-Adame, Semicond. Sci. Technol. 11, 1041 (1996).

${ }^{27}$ P. E. de Brito, C. A. A. da Silva, and H. N. Nazareno, Phys. Rev. B 51, 6096 (1995).

${ }^{28}$ F. Domínguez-Adame and E. Maciá, Phys. Lett. A 200, 69 (1995). 